\title{
Travelling and evanescent parts of the optical near field
}

\author{
HENK F. ARNOLDUS and JOHN T. FOLEY \\ Department of Physics and Astronomy, Mississippi State University, \\ PO Drawer 5167, Mississippi State, Mississippi 39762-5167, USA; \\ e-mails: arnoldus@ra.msstate.edu; jtf1@ra.msstate.edu
}

(Received 17 June 2002; revision received 15 October 2002)

\begin{abstract}
The optical near field of a localized source has been studied by means of the angular spectrum representation of the electromagnetic Green's tensor. This Green's tensor can be expressed in terms of four auxiliary functions, which depend on the field point through the dimensionless radial distance $q$ to the source, or origin of coordinates, and the polar angle $\theta$ with the $z$ axis. Each function separates into a part containing travelling (radiative) waves and a part which is a superposition of evanescent (decaying) waves. We have derived series expansions in $q$ of the four functions, both for the travelling and for the evanescent parts. It is shown that the travelling waves are finite at the origin of coordinates, and that all singular behaviour of the radiation field is governed by the evanescent waves. It is illustrated numerically that the series expansions are applicable up to about five wavelengths from the origin. In order to extend the range to also cover larger values of $q$, we have derived series expansions of the auxiliary functions which converge rapidly near the $x-y$ plane, and a full asymptotic expansion with the $z$ coordinate as the large variable. Finally, from the properties of the Taylor coefficients we have derived simple new integral representations for the auxiliary functions.
\end{abstract}

\section{Introduction}

Nanoscale technology appears to be emerging as one of the most promising new technological advances in recent years. In nanoscale structures and devices the spatial variations are well below an optical wavelength, and it has become imperative to study optical phenomena, and in particular radiation from atoms and molecules, on a similar length scale. This research area of near-field optics, or nano-optics, has evolved considerably over the last decade, in particular now that it has become feasible to measure light intensities within a sub wavelength range of the source with optical fibre tips [1-6]. At such short distances from the radiating atom or molecule, the electromagnetic field is dominated by the evanescent (decaying) modes, rather than by the propagating modes, which are usually observed in the far field with macroscopic devices. It has become experimentally and theoretically necessary to study the properties of evanescent radiation in the close vicinity of the source.

The appearance of evanescent waves in the radiation field of a localized source is most evident when the electric field is expressed as a superposition of transverse plane waves, known as the angular spectrum representation. For such a decomposition of the radiation field, one needs a reference surface, taken to be 
the $x-y$ plane, and usually this is a physical boundary such as a substrate on which molecules are deposited. Let $\boldsymbol{K}$ be the wave-vector of an individual partial wave of angular frequency $\omega$. For later convenience we shall indicate the magnitude of $\boldsymbol{K}$, the wavenumber, by $k_{0}$. The dispersion relation of free space then requires the relation $\omega=c k_{0}$. On the other hand, with $\boldsymbol{k}_{\|}$the component of $\boldsymbol{K}$ in the $x-y$ plane, assumed to be real valued and given, this determines the $z$ component of $\boldsymbol{K}$, apart from a minus sign, since $K_{z}^{2}=k_{0}^{2}-k_{\|}^{2}$. For $k_{\|}<k_{0}$ we have $K_{z}$ real, and therefore $\boldsymbol{K}$ is real. This situation corresponds to an ordinary travelling plane wave with wave-vector $\boldsymbol{K}$. On the other hand, when $k_{\|}>k_{0}$, the value of $K_{z}$ becomes imaginary. In this case, the wave still travels in the $x-y$ plane, in the direction of $\boldsymbol{k}_{\|}$ but now it decays exponentially in the $+z$ and $-z$ directions. These are the evanescent waves. In the angular spectrum representation, both types of wave appear simultaneously, with the evanescent waves dominating the near field whereas the travelling waves determine the radiation in the far field.

Traditionally, the far field has received most attention because of its relation to experimental observations. The radiative (far) field can be obtained in a very general way from an angular spectrum representation with the method of stationary phase $[7,8]$. This procedure yields an asymptotic approximation for the electromagnetic radiation field at large distances from the source, and it can be shown that this far field is determined by the travelling modes of the angular spectrum. An exception to this is when the observation direction is parallel to the $x-y$ plane, in which case the critical-point wave-vector $\boldsymbol{K}$ lies on the borderline in the $\boldsymbol{k}_{\|}$plane separating the travelling and evanescent waves in the angular spectrum (we then have $k_{\|}=k_{0}$ ). For this propagation direction, the evanescent waves do not decay and contribute equally to the far field, compared with the travelling waves. A second exception is for propagation perpendicular to the $x-y$ plane ( $\boldsymbol{K}$ along the $z$ axis). It can be shown that for this direction the evanescent waves also survive in the far field [9-11].

In this paper we present results for the travelling and evanescent parts of the near field, and we show explicitly how the near field at very short distances from the source is dominated by the evanescent waves. We also derive series expansions for larger distances, which are complementary to the near-field results. We show that there is a region of medium range where both approaches yield the same result.

\section{Angular spectrum}

The electric field $\boldsymbol{E}(\boldsymbol{r})$ of radiation emitted by a current density $\boldsymbol{j}(\boldsymbol{r})$ can be expressed most compactly with the aid of the electromagnetic Green's tensor $\mathbf{g}(\boldsymbol{r})$, according to $[12,13]$

$$
\boldsymbol{E}(\boldsymbol{r})=\frac{\mathrm{i} \omega \mu_{0}}{4 \pi} \int \mathrm{d} \boldsymbol{r}^{\prime} \mathbf{g}\left(\boldsymbol{r}-\boldsymbol{r}^{\prime}\right) \cdot \boldsymbol{j}\left(\boldsymbol{r}^{\prime}\right),
$$

in the Fourier angular frequency domain. We shall suppress the $\omega$ dependence of the various quantities throughout. When the source is an atom or molecule located at the origin of coordinates, then the current density represents the dipole moment $\boldsymbol{d}$, and equation (1) simplifies to

$$
\boldsymbol{E}(\boldsymbol{r})=\frac{k_{0}^{2}}{4 \pi \varepsilon_{0}} \mathbf{g}(\boldsymbol{r}) \cdot \boldsymbol{d}
$$


Hence all spatial dependence is incorporated in the Green's tensor. Therefore, rather than considering the field $\boldsymbol{E}(\boldsymbol{r})$ itself, we shall study the travelling and evanescent parts of the Green's tensor.

The representation of the Green's tensor in configuration space is given by [14]

$$
\mathbf{g}(\boldsymbol{r})=-\frac{4 \pi}{3 k_{0}^{2}} \delta(\boldsymbol{r}) \boldsymbol{I}+\left(\boldsymbol{I}+\frac{1}{k_{0}^{2}} \nabla \nabla\right) \frac{e^{\mathrm{i} k_{0} r}}{r},
$$

where $\mathbf{I}$ indicates the unit dyad. The term proportional to $\delta(\mathbf{r})$ gives a contribution to the field $\boldsymbol{E}(\boldsymbol{r})$, which is directly proportional to the current density at the same location, according to equation (1). This so-called self-field is sometimes neglected in the literature but, since this term could be considered as the ultimate near field, we shall retain it here. Also, it can be shown that this term has to be included for mathematical consistency $[15,16]$, especially when adopting transformations to reciprocal space or when employing angular spectrum representations [17]. Equation (3) for $\mathbf{g}(\boldsymbol{r})$ has, apart from the delta function, a singularity at $r=0$, and when substituted into equation (1) this leads to a singularity at $\boldsymbol{r}^{\prime}=\boldsymbol{r}$, which has to be integrated over when the field point $\boldsymbol{r}$ is inside the source region. It is understood that this integral is performed as a principal-value integral in the following manner; a small sphere with radius $\varepsilon$ and centred at $\boldsymbol{r}$ is excluded from the integration region and, after evaluating the integral, we take $\varepsilon \downarrow 0$. It can be shown that, if this limit is taken in a different way (by taking a cylinder instead of a sphere, for instance), then the delta function contribution in equation (3) might be different [18-21].

The angular spectrum representation of the Green's tensor $\mathbf{g}(\boldsymbol{r})$ follows from Weyl's representation of the scalar Green's function [22, 23]:

$$
\frac{e^{\mathrm{i} k_{0} r}}{r}=\frac{\mathrm{i}}{2 \pi} \int \mathrm{d} \boldsymbol{k}_{\|} \frac{1}{\beta} e^{\mathrm{i} \boldsymbol{K} \cdot \boldsymbol{r}} .
$$

Here, $\boldsymbol{K}=\boldsymbol{k}_{\|}+\beta \operatorname{sgn}(z) \boldsymbol{e}_{z}$, and parameter $\beta$ is defined as $\beta=\left(k_{0}^{2}-k_{\|}^{2}\right)^{1 / 2}$ for $k_{\|}<k_{0}$ and as $\beta=\mathrm{i}\left(k_{\|}^{2}-k_{0}^{2}\right)^{1 / 2}$ for $k_{\|}>k_{0}$. Then we substitute the right-hand side of (4) into (3) and work out the derivatives. This gives

$$
\mathbf{g}(\boldsymbol{r})=-\frac{4 \pi}{k_{0}^{2}} \delta(\boldsymbol{r}) \boldsymbol{e}_{z} \boldsymbol{e}_{z}+\frac{i}{2 \pi} \int \mathrm{d} \boldsymbol{k}_{\|} \frac{1}{\beta}\left(\mathbf{I}-\frac{1}{k_{0}^{2}} \boldsymbol{K} \boldsymbol{K}\right) e^{\mathrm{i} \boldsymbol{K} \cdot \boldsymbol{r}},
$$

which is the angular spectrum representation of $\mathbf{g}(\boldsymbol{r})$. As mentioned in the introduction, this superposition contains travelling waves ( $\beta$ real) and evanescent waves ( $\beta$ imaginary). The additional delta function comes from differentiating $\operatorname{sgn}(z)$ twice with respect to $z$. It should be noted that this delta function does not represent the self-field, since it is different from the delta function in the Green's tensor (3).

\section{Auxiliary functions}

Representation (5) is a two-dimensional integral representation, with the integration running over the $\boldsymbol{k}_{\|}$plane. Whether a wave is travelling or evanescent depends only on $k_{\|}$, the magnitude of $\boldsymbol{k}_{\|}$, so there is no need to retain the 
dependence of the partial waves on the direction of $\boldsymbol{k}_{\|}$. For simplicity of notation, we shall use dimensionless variables. In particular we set $\hat{\mathbf{g}}(\boldsymbol{r})=\mathbf{g}(\boldsymbol{r}) / k_{0}$. After integrating over the polar angle in the $\boldsymbol{k}_{\|}$plane, representation (5) takes the form

$$
\begin{aligned}
\hat{\mathbf{g}}(\boldsymbol{r})= & -\frac{4 \pi}{k_{0}^{3}} \delta(\boldsymbol{r}) \boldsymbol{e}_{z} \boldsymbol{e}_{z}+\frac{1}{2}\left(\mathbf{I}+\boldsymbol{e}_{z} \boldsymbol{e}_{z}\right) M_{\mathrm{a}}(q, \theta)+\frac{1}{2}\left(\mathbf{I}-\boldsymbol{e}_{z} \boldsymbol{e}_{z}-2 \hat{\boldsymbol{r}}_{\|} \hat{\boldsymbol{r}}_{\|}\right) M_{\mathrm{b}}(q, \theta) \\
& +\frac{1}{2} \operatorname{sgn}(z)\left(\hat{\boldsymbol{r}}_{\|} \boldsymbol{e}_{z}+\boldsymbol{e}_{z} \hat{\boldsymbol{r}}_{\|}\right) M_{\mathrm{c}}(q, \theta)+\frac{1}{2}\left(\mathbf{I}-3 \boldsymbol{e}_{z} \boldsymbol{e}_{z}\right) M_{\mathrm{d}}(q, \theta),
\end{aligned}
$$

with $\hat{\boldsymbol{r}}_{\|}$the radial unit vector in the $x-y$ plane, $q=k_{0} r$ the dimensionless distance between the field point $\boldsymbol{r}$ and the origin, and $\theta$ the polar angle of $\boldsymbol{r}$ with the $z$ axis. The four auxiliary functions $M_{\mathrm{i}}(q, \theta)$ contain the remaining integration over $k_{\|}$, and they are defined explicitly by

$$
\begin{aligned}
& M_{\mathrm{a}}(q, \theta)=\mathrm{i} \int_{0}^{\infty} \mathrm{d} \alpha \frac{\alpha}{\hat{\beta}} J_{0}(\alpha \rho) \mathrm{e}^{\mathrm{i} \hat{\beta}|\zeta|} \\
& M_{\mathrm{b}}(q, \theta)=-\mathrm{i} \int_{0}^{\infty} \mathrm{d} \alpha \frac{\alpha^{3}}{\hat{\beta}} J_{2}(\alpha \rho) \mathrm{e}^{\mathrm{i} \hat{\beta}|\zeta|} \\
& M_{\mathrm{c}}(q, \theta)=2 \int_{0}^{\infty} \mathrm{d} \alpha \alpha^{2} J_{1}(\alpha \rho) \mathrm{e}^{\mathrm{i} \hat{\beta}|\zeta|} \\
& M_{\mathrm{d}}(q, \theta)=\mathrm{i} \int_{0}^{\infty} \mathrm{d} \alpha \alpha \hat{\beta} J_{0}(\alpha \rho) \mathrm{e}^{\mathrm{i} \hat{\beta}|\zeta|}
\end{aligned}
$$

where $J_{\mathrm{n}}(x)$ are Bessel functions. Here we have introduced $\hat{\beta}=\beta / k_{0}$, the dimensionless $z$ coordinate $\zeta=k_{0} z$, which is $\zeta=q \cos \theta$, and $\rho=q \sin \theta$ as the dimensionless distance to the $z$ axis. The new integration variable is $\alpha=k_{\|} / k_{0}$, so that the integration range $[0,1)$ represents the superposition of travelling waves, and $1<\alpha<\infty$ covers the evanescent waves. The first two integrals have a singularity at $\alpha=1$ owing to the factor $1 / \hat{\beta}$, but this singularity is integrable from each side.

On the other hand, the Green's tensor is given by equation (3). When we work out the derivatives in this expression and use the notation $q=k_{0} r$, this becomes

$$
\hat{\mathbf{g}}(\boldsymbol{r})=-\frac{4 \pi}{3 k_{0}^{3}} \delta(\boldsymbol{r}) \mathbf{I}+\left[\left(1+\frac{\mathrm{i}}{q}-\frac{1}{q^{2}}\right) \mathbf{I}-\left(1+\frac{3 \mathrm{i}}{q}-\frac{3}{q^{2}}\right) \hat{\boldsymbol{r}} \hat{\boldsymbol{r}}\right] \frac{\mathrm{e}^{\mathrm{i} q}}{q},
$$

with $\hat{\boldsymbol{r}}=\boldsymbol{r} / r$. Obviously, this should be identical to equation (6) for $\hat{\mathbf{g}}(\boldsymbol{r})$. With $\hat{\boldsymbol{r}}=\hat{\boldsymbol{r}}_{\|} \sin \theta+\boldsymbol{e}_{z} \cos \theta$ we can compare the coefficients of the dyadic parts in both expressions. This leads to a set of four equations, which can be solved for the four functions $M_{\mathrm{i}}(q, \theta)$. We find that

$$
\begin{aligned}
& M_{\mathrm{a}}(q, \theta)=\frac{\mathrm{e}^{\mathrm{i} q}}{q} \\
& M_{\mathrm{b}}(q, \theta)=\sin ^{2} \theta\left(1+\frac{3 \mathrm{i}}{q}-\frac{3}{q^{2}}\right) \frac{\mathrm{e}^{\mathrm{i} q}}{q} \\
& M_{\mathrm{c}}(q, \theta)=-|\sin (2 \theta)|\left(1+\frac{3 \mathrm{i}}{q}-\frac{3}{q^{2}}\right) \frac{\mathrm{e}^{\mathrm{i} q}}{q}, \\
& M_{\mathrm{d}}(q, \theta)=-\frac{8 \pi}{3 k_{0}^{3}} \delta(\boldsymbol{r})+\left(\frac{1}{q}-\mathrm{i}\right) \frac{\mathrm{e}^{\mathrm{i} q}}{q^{2}}+\cos ^{2} \theta\left(1+\frac{3 \mathrm{i}}{q}-\frac{3}{q^{2}}\right) \frac{\mathrm{e}^{\mathrm{i} q}}{q} .
\end{aligned}
$$


We recognize $M_{\mathrm{a}}(q, \theta)$ as the scalar Green's function (4), apart from a factor $k_{0}$. The functions $M_{\mathrm{b}}(q, \theta)$ and $M_{\mathrm{c}}(q, \theta)$ have identical dependences on $q$, and they only differ in the overall angular dependence. Function $M_{\mathrm{d}}(q, \theta)$ has a delta function term and, when added to the delta function in the representation (6), we obtain exactly the self-field term in equation (3).

\section{The splitting}

Each of the four functions has a travelling and an evanescent part, and with equation (6) this determines the travelling part $\hat{\mathbf{g}}(\boldsymbol{r})^{\mathrm{tr}}$ and evanescent part $\hat{\mathbf{g}}(\boldsymbol{r})^{\mathrm{ev}}$ of the Green's tensor. It should be noted that the delta functions in equations (5) and (6) are neither travelling nor evanescent since only the integral over $\boldsymbol{k}_{\|}$in equation (5) splits into travelling and evanescent components. So the Green's tensor can be written as

$$
\hat{\mathbf{g}}(\boldsymbol{r})=-\frac{4 \pi}{k_{0}^{3}} \delta(\boldsymbol{r}) \boldsymbol{e}_{z} \boldsymbol{e}_{z}+\hat{\mathbf{g}}(\boldsymbol{r})^{\mathrm{tr}}+\hat{\mathbf{g}}(\boldsymbol{r})^{\mathrm{ev}}
$$

For the evanescent parts we restrict the integration ranges in equations (7)-(10) to $1<\alpha<\infty$. We then note that the parameter $\hat{\beta}$ is imaginary, and all factors of $\mathrm{i}$ cancel. Therefore, the evanescent waves are pure real. Vice versa, this implies that the imaginary part of the Green's tensor consists entirely of travelling waves. When we take the imaginary parts of equations (12)-(15) we find explicitly that

$$
\begin{aligned}
& \operatorname{Im}\left[M_{\mathrm{a}}(q, \theta)\right]=\frac{\sin q}{q} \\
& \operatorname{Im}\left[M_{\mathrm{b}}(q, \theta)\right]=-\frac{\sin ^{2} \theta}{q}\left[\left(\frac{3}{q^{2}}-1\right) \sin q-\frac{3 \cos q}{q}\right] \\
& \operatorname{Im}\left[M_{\mathrm{c}}(q, \theta)\right]=\frac{|\sin (2 \theta)|}{q}\left[\left(\frac{3}{q^{2}}-1\right) \sin q-\frac{3 \cos q}{q}\right] \\
& \operatorname{Im}\left[M_{\mathrm{d}}(q, \theta)\right]=\cos ^{2} \theta \frac{\sin q}{q}+\left(1-3 \cos ^{2} \theta\right) \frac{1}{q^{2}}\left(\frac{\sin q}{q}-\cos q\right) .
\end{aligned}
$$

From the viewpoint of the near field, we should consider these functions in the neighbourhood of $q=0$. With a Taylor expansion it follows easily that all four contributions are finite at $q=0$. More precisely, $\operatorname{Im}\left[M_{\mathrm{a}}(q, \theta)\right]=1+\mathrm{O}\left(q^{2}\right)$, $\operatorname{Im}\left[M_{\mathrm{d}}(q, \theta)\right]=1 / 3+\mathrm{O}\left(q^{2}\right)$, and the other two are both $\mathrm{O}\left(q^{2}\right)$ around $q=0$. The corresponding part of the Green's tensor is then

$$
\operatorname{Im}\left[\hat{\mathbf{g}}(\boldsymbol{r})^{\operatorname{tr}}\right]=\frac{2}{3} \mathbf{I}+\mathrm{O}\left(q^{2}\right)
$$

It will be shown below that the real part of $\hat{\mathbf{g}}(\boldsymbol{r})^{\operatorname{tr}}$ is $\mathrm{O}(\mathrm{q})$; so the first term on the right-hand side of equation (21) also gives the leading term in the near field of the total travelling part, in agreement with [9].

Since the imaginary parts of functions $M_{\mathrm{i}}(q, \theta)$ are pure travelling, we only need to consider the splitting of the real parts. When we take the real part of equation (7), restrict the integration range to $0 \leq \alpha<1$ and make a change in 
variables according to $u=\left(1-\alpha^{2}\right)^{1 / 2}$, we obtain the representation

$$
\operatorname{Re}\left[M_{\mathrm{a}}(q, \theta)^{\operatorname{tr}}\right]=-\int_{0}^{1} \mathrm{~d} u J_{0}\left[\rho\left(1-u^{2}\right)^{1 / 2}\right] \sin (u|\zeta|) .
$$

The advantage of the change in variables is that the singularity in the integrand near $\alpha=1$ has disappeared. The dependence on the spatial coordinates $q$ and $\theta$ enters through $\rho=q \sin \theta$ in the argument of the Bessel function and through $\zeta=q \cos \theta$. For the evanescent part we set $u=\left(\alpha^{2}-1\right)^{1 / 2}$, which then gives

$$
M_{\mathrm{a}}(q, \theta)^{\mathrm{ev}}=\int_{0}^{\infty} \mathrm{d} u J_{0}\left[\rho\left(1+u^{2}\right)^{1 / 2}\right] \mathrm{e}^{-u|\zeta|} .
$$

Similar expressions hold for the other three integrals. Owing to the complicated appearance of $q$ and $\theta$ it is not obvious what the behaviour of equations (22) and (23) is near the origin.

\section{Travelling part}

In order to reveal the near-field properties of $\operatorname{Re}\left[M_{\mathrm{a}}(q, \theta)^{\mathrm{tr}}\right]$, we make a Taylor expansion of equation (22) in $q$, with $\theta$ considered fixed. To this end, we expand the Bessel function and the sine in their known series. This leads to a double series, which can be reorganized in a single series by means of the Cauchy product. Next we integrate term by term over $u$ and collect the powers of $q$, which then yields the series expansion

$$
\operatorname{Re}\left[M_{a}(q, \theta)^{\mathrm{tr}}\right]=-\frac{1}{2} q \sum_{n=0}^{\infty} A_{n}(\theta) \frac{\left(-\frac{1}{4} q^{2}\right)^{n}}{n !(n+1) !} .
$$

The coefficient functions $A_{n}(\theta)$ are found to be

$$
A_{n}(\theta)=|\cos \theta| n ! \sum_{m=0}^{n} \frac{m !}{(n-m) !(2 m+1) !}\left(\sin ^{2} \theta\right)^{n-m}\left(4 \cos ^{2} \theta\right)^{m} .
$$

Along the same lines we obtain the Taylor series for $\operatorname{Re}\left[M_{\mathrm{b}}(q, \theta)^{\operatorname{tr}}\right]$ :

$$
\operatorname{Re}\left[M_{\mathrm{b}}(q, \theta)^{\mathrm{tr}}\right]=\frac{1}{8} q^{3} \sin ^{2} \theta \sum_{n=0}^{\infty} A_{n}(\theta) \frac{\left(-\frac{1}{4} q^{2}\right)^{n}}{n !(n+3) !} .
$$

It seems remarkable that the coefficient functions $A_{n}(\theta)$ for this expansion are identical with those for the expansion of $\operatorname{Re}\left[M_{\mathrm{a}}(q, \theta)^{\operatorname{tr}}\right]$. For the third function we find that

$$
\operatorname{Re}\left[M_{\mathrm{c}}(q, \theta)^{\operatorname{tr}}\right]=\frac{1}{2} q \sin \theta \sum_{n=0}^{\infty} C_{n}(\theta) \frac{\left(-\frac{1}{4} q^{2}\right)^{n}}{n !(n+2) !},
$$

and this involves a slightly different coefficient function:

$$
C_{n}(\theta)=n ! \sum_{m=0}^{n} \frac{m !}{(n-m) !(2 m) !}\left(\sin ^{2} \theta\right)^{n-m}\left(4 \cos ^{2} \theta\right)^{m} .
$$


It can be verified from equations (28) and (25) that the functions $C_{n}(\theta)$ are related to the functions $A_{n}(\theta)$ according to

$$
C_{n}(\theta)=(\sin \theta)^{2 n}+2 n|\cos \theta| A_{n-1}(\theta), \quad n=1,2, \ldots
$$

The expansion of the last function is given by

$$
\operatorname{Re}\left[M_{\mathrm{d}}(q, \theta)^{\mathrm{tr}}\right]=-\frac{1}{4} q \sum_{n=0}^{\infty}\left[A_{n}(\theta)+|\cos \theta| C_{n}(\theta)\right] \frac{\left(-\frac{1}{4} q^{2}\right)^{n}}{n !(n+2) !}
$$

which does not involve any new coefficient functions.

The leading order in $q$ follows by retaining only the $n=0$ term. With $A_{0}(\theta)=|\cos \theta|$ and $C_{0}(\theta)=1$ we then see that $\operatorname{Re}\left[M_{\mathrm{a}}(q, \theta)^{\operatorname{tr}}\right]=-(q / 2)|\cos \theta|$, $\operatorname{Re}\left[M_{\mathrm{c}}(q, \theta)^{\operatorname{tr}}\right]=(q / 4) \sin \theta, \quad \operatorname{Re}\left[M_{\mathrm{d}}(q, \theta)^{\operatorname{tr}}\right]=-(q / 4)|\cos \theta|, \quad$ and $\operatorname{Re}\left[M_{\mathrm{b}}(q, \theta)^{\operatorname{tr}}\right]=$ $\mathrm{O}\left(q^{3}\right)$. When we use this in the travelling part of equation (6) and rearrange the tensorial parts we find that

$$
\operatorname{Re}\left[\hat{\mathbf{g}}(\boldsymbol{r})^{\operatorname{tr}}\right]=\frac{1}{8} q \operatorname{sgn}(\zeta)\left[\hat{\boldsymbol{r}} \boldsymbol{e}_{z}+\boldsymbol{e}_{z} \hat{\boldsymbol{r}}-3 \mathbf{I}-\boldsymbol{e}_{z} \boldsymbol{e}_{z}\right]+\mathrm{O}\left(q^{3}\right)
$$

This shows that the real part of the travelling waves in the near field is indeed $\mathrm{O}(q)$, and equation (31) gives the exact appearance.

The real part of $M_{\mathrm{a}}(q, \theta)^{\mathrm{tr}}$ is shown in figure 1 , for $\theta=30^{\circ}$. It is illustrated that, for reasonably small $q$, only a few terms already reproduce the exact value (obtained by numerical integration) and, when more terms are retained, not only does the approximation become more accurate, but also the range of $q$ values where the series expansion is applicable increases. It should be noted that the series (24) converges for all $q$, but that numerically there is a limit on the number of terms that can be summed owing to machine accuracy. We have found that, in double precision, the series converges up to about $q=35$ when 50 terms are used. After that, numerical instabilities set in, and other methods for evaluating this function

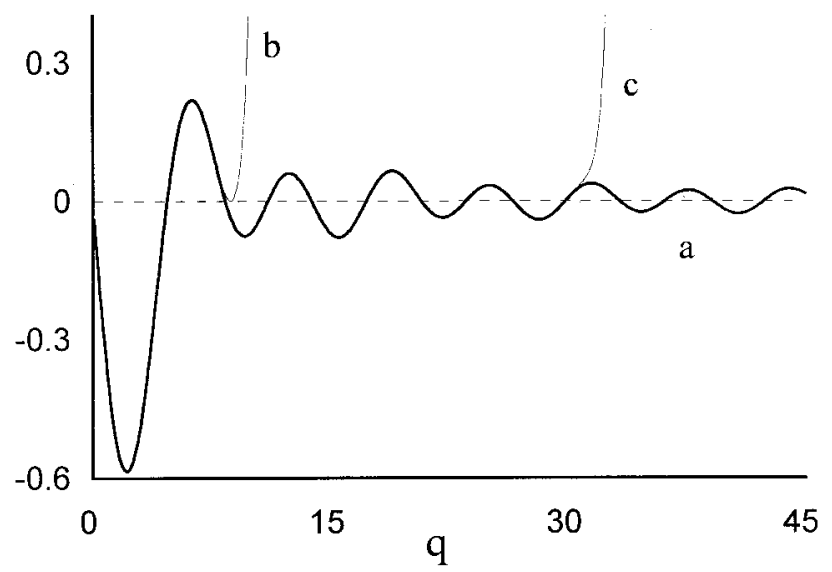

Figure 1. Illustration of the exact value of $\operatorname{Re}\left[M_{\mathrm{a}}(q, \theta)^{\mathrm{tr}}\right]$ (curve a) and its approximation by ten terms in the series expansion (24) (curve b), as functions of $q$ and for $\theta=30^{\circ}$. Curve c shows the improvement when 40 terms in equation (24) are used. 
have to be employed. In any case, for the near field this series expansion appears to be more than adequate. We have also verified that, for the other three functions, similar conclusions hold.

\section{Coefficient functions $A_{\boldsymbol{n}}(\boldsymbol{\theta})$}

The expansion coefficients $A_{n}(\theta)$, defined by equation (25), can be simplified as follows. We introduce a generating function

$$
\chi(\theta ; t)=\sum_{n=0}^{\infty} A_{n}(\theta) \frac{t^{n}}{n !} .
$$

After substitution of equation (25), this series has the form of a Cauchy product and can be written as the product of two series, both of which can be evaluated in closed form. We then obtain

$$
\chi(\theta ; t)=\frac{1}{2}\left(\frac{\pi}{t}\right)^{1 / 2} \mathrm{e}^{t} \operatorname{erf}\left(|\cos \theta| t^{1 / 2}\right), \quad t>0
$$

Conversely, the coefficient functions $A_{n}(\theta)$ follow from the generating function according to

$$
A_{n}(\theta)=\left.\frac{\mathrm{d}^{n}}{\mathrm{~d} t^{n}} \chi(\theta ; t)\right|_{t=0} .
$$

With the known series expansion of the error function and term-by-term differentiation this yields the alternative simpler form for $A_{n}(\theta)$ :

$$
A_{n}(\theta)=n ! \sum_{m=0}^{n} \frac{(-1)^{m}}{(n-m) ! m !(2 m+1)}|\cos \theta|^{2 m+1} .
$$

It can then be verified by inspection that $A_{n}(\theta)$ can be expressed in terms of a hypergeometric function according to $A_{n}(\theta)=|\cos \theta|{ }_{2} F_{1}\left(\frac{1}{2},-n, \frac{3}{2} ; \cos ^{2} \theta\right)$.

Some interesting properties of $A_{n}(\theta)$ can be derived from this representation. Firstly we notice that the $\theta$ dependence only enters through $|\cos \theta|$. We temporarily set $x=|\cos \theta|$, and write $Y_{n}(x)$ for the right-hand side of equation (35). We then see immediately from equation (35) that the derivative of $Y_{n}(x)$ is

$$
\frac{\mathrm{d} Y_{n}(x)}{\mathrm{d} x}=\left(1-x^{2}\right)^{n}
$$

and therefore

$$
Y_{n}(x)=\int_{0}^{x} \mathrm{~d} t\left(1-t^{2}\right)^{n}
$$

since $Y_{n}(0)=0$. With some further manipulations and integration by parts it can be shown from this representation that the functions $Y_{n}(x)$ satisfy the following recurrence relation:

$$
(2 n+1) Y_{n}(x)=2 n Y_{n-1}(x)+x\left(1-x^{2}\right)^{n} .
$$


Then we set again $x=|\cos \theta|$, and with $Y_{n}(|\cos \theta|)=A_{n}(\theta)$ we obtain

$$
A_{n}(\theta)=\frac{1}{2 n+1}\left[2 n A_{n-1}(\theta)+|\cos \theta|(\sin \theta)^{2 n}\right], \quad n=1,2, \ldots
$$

This relation determines all $A_{n}(\theta)$ recursively from the first value $A_{0}(\theta)=|\cos \theta|$. From a numerical point of view, this method is far superior to repeatedly computing the sum in equation (35).

For the series expansion of all four auxiliary functions, we also need the coefficient functions $C_{n}(\theta)$. After generating the functions $A_{n}(\theta)$ with the recursion (39), the functions $C_{n}(\theta)$ follow from equation (29). On the other hand, since both functions are related by equation (29), a recursion between the functions $A_{n}(\theta)$ implies a recursion between the functions $C_{n}(\theta)$. This recurrence relation is found to take the form

$$
(2 n-1) C_{n}(\theta)=2 n C_{n-1}(\theta)-(\sin \theta)^{2 n}, \quad n=1,2, \ldots,
$$

and with $C_{0}(\theta)=1$ this determines all $C_{n}(\theta)$.

Some other features of $A_{n}(\theta)$ can be derived from the results above. In equation (39), the right-hand side is non-negative if $A_{n-1}(\theta) \geq 0$. Since $A_{0}(\theta) \geq 0$, we conclude that $A_{n}(\theta) \geq 0$ for all $n$. On the other hand, if $A_{n-1}(\theta) \leq 1$, then the factor in square brackets does not exceed $2 n+1$, and therefore we have $A_{n}(\theta) \leq 1$. Furthermore, we see from equation (35) that $A_{n}(\pi / 2)=0$. To find $A_{n}(0)$, we go back to the integral representation (37). For $x=1$ this integral can be evaluated in closed form:

$$
A_{n}(0)=\int_{0}^{1} \mathrm{~d} t\left(1-t^{2}\right)^{n}=\frac{4^{n}(n !)^{2}}{(2 n+1) !} .
$$

If we set $\theta=\pi / 2$ in equation (29), we obtain $C_{n}(\pi / 2)=1$ for all $n$. On the other hand, with $\theta=0$ this relation gives $C_{n}(0)=2 n A_{n-1}(0)$, and with equation (41) this becomes

$$
C_{n}(0)=\frac{4^{n}(n !)^{2}}{(2 n) !}
$$

In order to see the dependence on $n$ for $n$ large, we use Stirling's formula to approximate the factorials. From equations (41) and (42) we then derive

$$
\begin{aligned}
& A_{n}(0) \approx \frac{1}{2}\left(\frac{\pi}{n}\right)^{1 / 2}, \\
& C_{n}(0) \approx(\pi n)^{1 / 2},
\end{aligned}
$$

both for $n$ large. This shows that the coefficient functions have only a very mild $n$ dependence, with the $A_{n}(\theta)$ values decreasing and the $C_{n}(\theta)$ values increasing. Figure 2 shows a typical example of the coefficient functions as a function of $\theta$. 


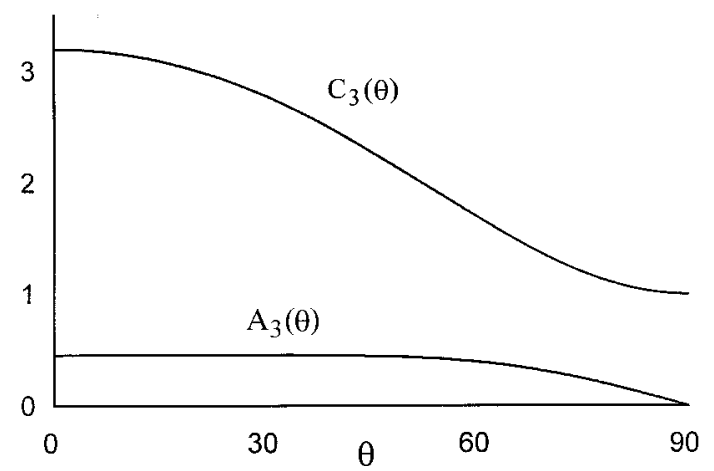

Figure 2. Coefficient functions $A_{3}(\theta)$ and $C_{3}(\theta)$ as a functions of $\theta$ (in degrees).

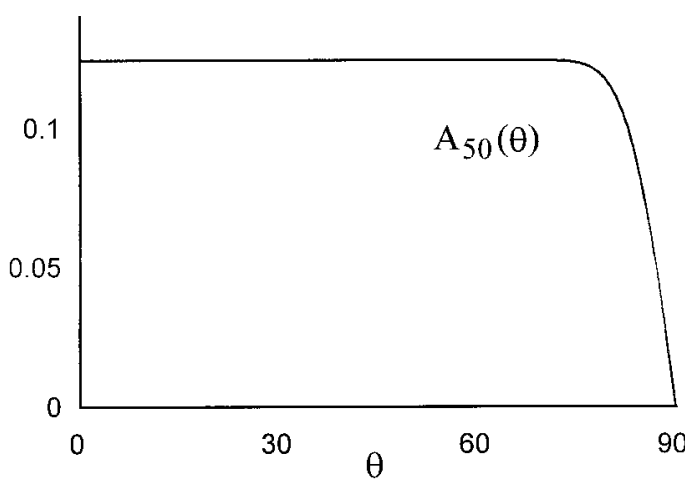

Figure 3. Graph of $A_{50}(\theta)$, illustrating that the dependence of this function on $\theta$ is very weak, provided that $\theta<90^{\circ}$.

As for the $\theta$ dependence, an interesting feature of the functions $A_{n}(\theta)$ can be derived as follows. Equation (37) is an integral of equation (36), but we can equally integrate equation (36) as, with $x=|\cos \theta|$,

$$
A_{n}(\theta)=A_{n}(0)-\int_{|\cos \theta|}^{1} \mathrm{~d} t\left(1-t^{2}\right)^{n},
$$

with $A_{n}(0)$ given by equation (41). For $n$ sufficiently large, we have $A_{n}(0) \propto n^{-1 / 2}$, but the second term on the right-hand side of equation (45) vanishes exponentially with $n$, provided that the lower limit does not approach zero. Therefore, we have $A_{n}(\theta) \approx A_{n}(0)$ for $n$ large, and $\theta$ not too close to $\pi / 2$. Figure 3 shows $A_{50}(\theta)$, and from the data we have found that the curve is flat in six figures up to $\theta=64^{\circ}$. We see from figure 2 that already for $n=3$ the $\theta$ dependence of $A_{3}(\theta)$ is very weak, and from the data it shows that up to $36^{\circ}$ the value of $A_{3}(\theta)$ is constant in the two figures.

\section{Evanescent part}

In order to obtain the series expansion of the evanescent part of $\hat{\mathbf{g}}(\boldsymbol{r})$ near the origin of coordinates, we use the fact that the sum of the evanescent part and the travelling part of each auxiliary function is known and given by the real parts of equations (12)-(15). We expand each real part in a Taylor series in $q$, and then take 
the difference from the series for the travelling parts, as given in section 5 . Then we combine the series by grouping the powers of $q$. For the first function we obtain in this fashion

$$
M_{\mathrm{a}}(q, \theta)^{\mathrm{ev}}=\frac{1}{q}-\frac{1}{2} q \sum_{n=0}^{\infty} a_{n}(\theta) \frac{\left(-\frac{1}{4} q^{2}\right)^{n}}{n !(n+1) !} .
$$

The first term, $1 / q$, diverges at the origin of coordinates, which indicates that for $q$ sufficiently small this term will dominate the remainder of the series in equation (46), and the travelling waves, since these are finite for $q=0$. The series in equation (46) is, apart from the $1 / q$, identical in form with the series $(24)$ for $\operatorname{Re}\left[M_{\mathrm{a}}(q, \theta)^{\mathrm{tr}}\right]$, with $A_{n}(\theta)$ replaced by $a_{n}(\theta)$. Furthermore, we find that the new coefficient functions are related to the previous coefficient functions by the simple relation

$$
a_{n}(\theta)=A_{n}(0)-A_{n}(\theta) .
$$

Comparison with equation (45) shows that $a_{n}(\theta)$ has the integral representation

$$
a_{n}(\theta)=\int_{|\cos \theta|}^{1} \mathrm{~d} t\left(1-t^{2}\right)^{n}
$$

which we shall use in section 10. Furthermore, equation (39) implies a recursion relation for the coefficients $a_{n}(\theta)$ :

$$
a_{n}(\theta)=\frac{1}{2 n+1}\left[2 n a_{n-1}(\theta)-|\cos \theta|(\sin \theta)^{2 n}\right],
$$

with initial value $a_{0}(\theta)=1-|\cos \theta|$. Figure 4 shows $M_{\mathrm{a}}(q, \theta)^{\mathrm{ev}}$ and its series representation.

For the second function we find that

$$
M_{\mathrm{b}}(q, \theta)^{\mathrm{ev}}=-\sin ^{2} \theta\left(\frac{3}{q^{3}}+\frac{1}{2 q}+\frac{1}{8} q\right)+\frac{1}{8} q^{3} \sin ^{2} \theta \sum_{n=0}^{\infty} a_{n}(\theta) \frac{\left(-\frac{1}{4} q^{2}\right)^{n}}{n !(n+3) !}
$$

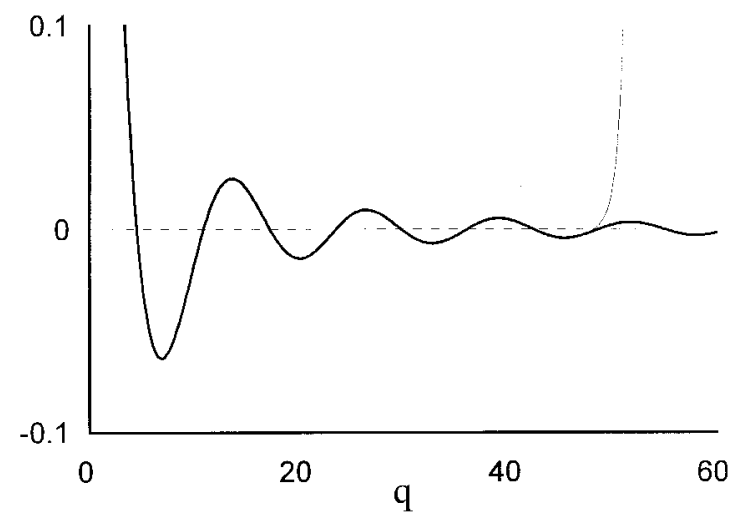

Figure 4. The exact $M_{\mathrm{a}}(q, \theta)^{\mathrm{ev}}$ from equation (23) for $\theta=30^{\circ}(-)$ and its approximation (-) by 40 terms in the series expansion (46). 
and, just as for the travelling part, the coefficient functions $a_{n}(\theta)$ are the same as for $M_{\mathrm{a}}(q, \theta)^{\mathrm{ev}}$. Also, the second part in equation (50) has the same appearance as the series (26). Two singular terms, $\mathrm{O}\left(q^{-3}\right)$ and $\mathrm{O}\left(q^{-1}\right)$, precede the Taylor series, and an additional linear term appears. The remaining two functions are found to be

$$
\begin{aligned}
M_{\mathrm{c}}(q, \theta)^{\mathrm{ev}}= & |\sin (2 \theta)|\left(\frac{3}{q^{3}}+\frac{1}{2 q}\right)+\frac{1}{2} q \sin \theta \sum_{n=0}^{\infty} c_{n}(\theta) \frac{\left(-\frac{1}{4} q^{2}\right)^{n}}{n !(n+2) !}, \\
M_{\mathrm{d}}(q, \theta)^{\mathrm{ev}}= & -\frac{8 \pi}{3 k_{0}^{3}} \delta(\boldsymbol{r})+\left(1-3 \cos ^{2} \theta\right) \frac{1}{q^{3}}+\sin ^{2} \theta \frac{1}{2 q} \\
& -\frac{1}{4} q \sum_{n=0}^{\infty}\left[a_{n}(\theta)+|\cos \theta| c_{n}(\theta)\right] \frac{\left(-\frac{1}{4} q^{2}\right)^{n}}{n !(n+2) !},
\end{aligned}
$$

which involve the new coefficient functions $c_{n}(\theta)$, defined by

$$
c_{n}(\theta)=|\cos \theta| C_{n}(0)-C_{n}(\theta)
$$

They obey the recursion relation

$$
(2 n-1) c_{n}(\theta)=2 n c_{n-1}(\theta)+(\sin \theta)^{2 n},
$$

with initial value $c_{0}(\theta)=|\cos \theta|-1$.

When we combine the evanescent parts of the four functions as in equation (6), we obtain for the evanescent part of the Green's tensor

$$
\hat{\mathbf{g}}(\boldsymbol{r})^{\mathrm{ev}}=-\frac{4 \pi}{3 k_{0}^{3}} \delta(\boldsymbol{r})\left(\mathbf{I}-3 \boldsymbol{e}_{z} \boldsymbol{e}_{z}\right)+\frac{1}{q^{3}}(3 \hat{\boldsymbol{r}} \hat{\boldsymbol{r}}-\mathbf{I})+\frac{1}{2 q}(\hat{\boldsymbol{r}} \hat{\boldsymbol{r}}+\mathbf{I})+\mathrm{O}(q) .
$$

It is interesting to note that $\hat{\mathbf{g}}(\boldsymbol{r})^{\mathrm{ev}}$ has a $q^{-3}$ contribution and a $q^{-1}$ contribution, but no $q^{-2}$ part. The total Green's tensor $\hat{\mathbf{g}}(\boldsymbol{r})$, given by equation (11), has $q^{-3}, q^{-2}$ and $q^{-1}$ terms and, for $q$ large, these terms represent the familiar near-, middleand far-field parts of the corresponding electric field. For small $q$, however, we need to expand $\mathrm{e}^{\mathrm{i} q}$ in equation (11) around $q=0$ and next collect powers of $q$. It can then be checked by inspection that this gives the right-hand side of equation (55), with the $q^{-2}$ term cancelling exactly. Obviously, this is the case since the travelling part is $\mathrm{O}(1)$ near the origin. Therefore, all singular behaviour near the origin, or near the source, arises because of the presence of evanescent waves, and equation (55) shows explicitly their appearance.

\section{Series expansion for small $\zeta$}

We have shown that the optical near field has distinctive contributions from travelling and evanescent waves, and we have shown how the separate parts of the Green's tensor can be obtained by series expansion in $q$. For computational purposes we also need to be able to evaluate the field for large $q$. This can be done by asymptotic expansion of $\hat{\mathbf{g}}(\boldsymbol{r})$ with the method of stationary phase. In this approach, however, the separate contributions from the travelling and evanescent waves are not resolved, and only the leading term of the far field is recovered. In this section we derive a series expansion which can be applied to evaluate the 
travelling and evanescent parts for large $q$. To this end we adopt dimensionless cylinder coordinates $(\rho, \zeta)$, with $\rho=q \sin \theta$ and $\zeta=q \cos \theta$, as in section 4 . We substitute equation (25) for $A_{n}(\theta)$ in equation (24) and change the order of summation. Next we collect powers of $|\zeta|$. It then appears that the coefficients in the series can be expressed in terms of Bessel functions, and the result is

$$
\operatorname{Re}\left[M_{\mathrm{a}}(q, \theta)^{\mathrm{tr}}\right]=\frac{1}{2} \sum_{k=0}^{\infty} \frac{k !}{(2 k+1) !}\left(-\frac{2}{\rho}\right)^{k+1} J_{k+1}(\rho)|\zeta|^{2 k+1} .
$$

In this fashion we obtain a Taylor series in $|\zeta|$, rather than $q$, and the coefficients are functions of $\rho$, rather than $\theta$. Since $J_{k+1}(\rho)=\mathrm{O}\left(\rho^{k+1}\right)$ near $\rho=0$, the negative powers of $\rho$ do not represent a singularity near the origin. More precisely, with the limit

$$
\lim _{\rho \rightarrow 0}\left[\left(\frac{2}{\rho}\right)^{k+1} J_{k+1}(\rho)\right]=\frac{1}{(k+1) !}
$$

the series (56) can be summed for $\rho=0$, and we find that

$$
\operatorname{Re}\left[M_{\mathrm{a}}(q, 0)^{\mathrm{tr}}\right]=\frac{1}{q}(\cos q-1)
$$

and the same for $\theta=\pi$.

Since equation (56) is a Taylor series in $|\zeta|$, we expect that its application is limited to small values of $|\zeta|$, which is the neighbourhood of the $x-y$ plane, although it can be shown that the series converges for all $\rho$ and $\zeta$. By retaining only the first term in the series we obtain the approximation

$$
\operatorname{Re}\left[M_{\mathrm{a}}(q, \theta)^{\mathrm{tr}}\right] \approx-\frac{1}{\rho} J_{l}(\rho)|\zeta|
$$

Figure 5 shows this approximation and the exact value as a function of $\rho$ and for $\zeta=1.5$. It appears that, for $\rho$ not too small, this approximation is excellent, even

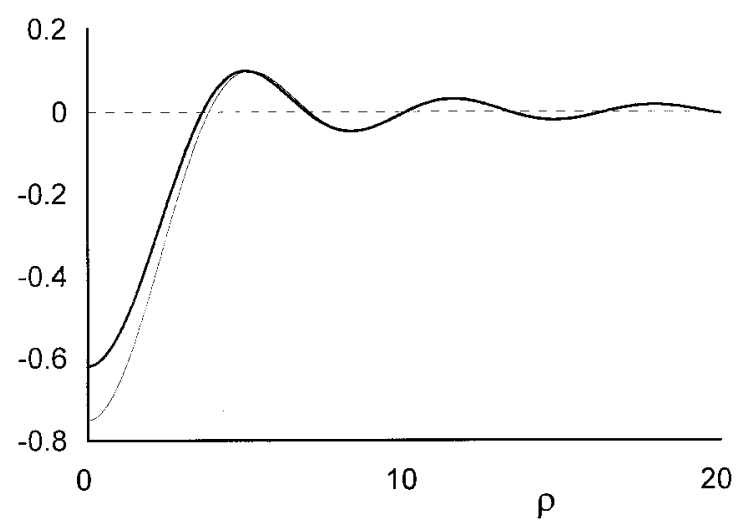

Figure 5. $\operatorname{Re}\left[M_{\mathrm{a}}(q, \theta)^{\mathrm{tr}}\right]$ as a function of $\rho$, for $\zeta=1.5(-)$ and its approximation (一) given by equation (59). 
though the value of $\zeta$ is not really small. For smaller values of $\zeta$ the difference between the exact value and approximation (59) disappears within drawing accuracy. We also conclude from the figure that the approximation improves with increasing $\rho$. Therefore, expansion (56) can be used for large values of $q$, provided that the field point is reasonably close to the $x-y$ plane.

The series expansions for the other three functions follow similarly, with the results

$$
\begin{gathered}
\operatorname{Re}\left[M_{\mathrm{b}}(q, \theta)^{\mathrm{tr}}\right]=-\frac{1}{2} \sum_{k=0}^{\infty} \frac{k !}{(2 k+1) !}\left(-\frac{2}{\rho}\right)^{k+1} J_{k+3}(\rho)|\zeta|^{2 k+1}, \\
\operatorname{Re}\left[M_{\mathrm{c}}(q, \theta)^{\operatorname{tr}}\right]=-\sum_{k=0}^{\infty} \frac{k !}{(2 k) !}\left(-\frac{2}{\rho}\right)^{k+1} J_{k+2}(\rho)|\zeta|^{2 k}, \\
\operatorname{Re}\left[M_{\mathrm{d}}(q, \theta)^{\mathrm{tr}}\right]=-\frac{1}{2} \sum_{k=0}^{\infty} \frac{(k+1) !}{(2 k+1) !}\left(-\frac{2}{\rho}\right)^{k+2} J_{k+2}(\rho)|\zeta|^{2 k+1} .
\end{gathered}
$$

On the $z$ axis we have from equations (60) and (61)

$$
\operatorname{Re}\left[M_{\mathrm{b}}(q, 0)^{\mathrm{tr}}\right]=\operatorname{Re}\left[M_{\mathrm{c}}(q, 0)^{\mathrm{tr}}\right]=0,
$$

since the orders of the Bessel functions are higher than the preceding inverse powers of $\rho$. For equation (62) we have to take the limit $\rho \rightarrow 0$ with equation (57). The remaining series can be summed in closed form, yielding

$$
\operatorname{Re}\left[M_{\mathrm{d}}(q, 0)^{\mathrm{tr}}\right]=\frac{2}{q^{3}}(1-\cos q)-\frac{2 \sin q}{q^{2}}+\frac{\cos q}{q} .
$$

Another result which can be found immediately from these series expansions is the value of the four functions for a field point in the $x-y$ plane. From equation (61) we have

$$
\operatorname{Re}\left[M_{\mathrm{c}}(q, \pi / 2)^{\mathrm{tr}}\right]=\frac{2}{q} J_{2}(q)
$$

and the other three functions vanish identically.

\section{Asymptotic expansion in $\zeta$}

Although the series from the previous section converge for all $|\zeta|$ and $\rho$, in practice the application is limited to small values of $|\zeta|$. It appears possible to also derive a series expansion which holds for $|\zeta|$ large and $\rho$ fixed. To this end, we start from the integral representations for the evanescent parts, as in equation (23). Noting that such integrals have the form of a Laplace transform, successive terms in the asymptotic series can be found by repeated integration by parts. The first two terms of the expansion of equation (23) were reported in [24], and in [11] the leading terms in the expansion of the evanescent parts of the four auxiliary functions (12)-(15) were reported. In this approach, one obtains an expansion that 
becomes invalid near the $x-y$ plane. With a more sophisticated method, a uniform expansion can be derived which holds also near the $x-y$ plane [25, 26]. The limitation of this approach is that only the leading term can be found. In this section we shall adopt a different method, leading to full asymptotic series for the evanescent parts of the four auxiliary functions, which generalizes the results of [24] and [11].

First we expand the Bessel function $J_{0}(x)$ in equation (23) in a Taylor series around $x=0$, which yields the representation

$$
M_{\mathrm{a}}(q, \theta)^{\mathrm{ev}}=\sum_{n=0}^{\infty} \frac{\left(-\frac{1}{4} \rho^{2}\right)^{n}}{(n !)^{2}} \int_{0}^{\infty} \mathrm{d} u\left(1+u^{2}\right)^{n} \mathrm{e}^{-u|\zeta|} .
$$

Then we expand $\left(1+u^{2}\right)^{n}$ with Newton's binomium and integrate term by term, leading to

$$
M_{\mathrm{a}}(q, \theta)^{\mathrm{ev}}=\sum_{n=0}^{\infty} \frac{\left(-\frac{1}{4} \rho^{2}\right)^{n}}{n !} \sum_{k=0}^{n} \frac{(2 k) !}{k !(n-k) !} \frac{1}{|\zeta|^{2 k+1}} .
$$

After changing the order of summation, we recognize the series expansion of Bessel functions of order $k$. Finally this gives

$$
M_{\mathrm{a}}(q, \theta)^{\mathrm{ev}}=\sum_{k=0}^{\infty} \frac{(2 k) !}{k !}\left(-\frac{1}{2} \rho\right)^{k} J_{k}(\rho) \frac{1}{|\zeta|^{2 k+1}} .
$$

It is interesting to note the resemblence with the expansion (56). Obviously, for $\zeta \rightarrow 0$ the series expansion (68) has no meaning. The result (68) is to be understood as an asymptotic series with $|\zeta|$ as the large parameter and $\rho$ fixed. The two leading terms are

$$
M_{\mathrm{a}}(q, \theta)^{\mathrm{ev}}=\mathcal{F}_{0}(\rho) \frac{1}{|\zeta|}-\rho J_{1}(\rho) \frac{1}{|\zeta|^{3}}+\cdots,
$$

in agreement with earlier results. This asymptotic expansion is extremely accurate, even for $|\zeta|$ or $q$ relatively small. We have drawn a graph of the first term in equation (69) for $\theta=30^{\circ}$, and it appeared that $J_{0}(\rho) /|\zeta|$ is indistinguishable from the exact solution within graphing resolution. Only for $|\zeta| \lesssim 2 \pi$ (the $z$ coordinate smaller that about a wavelength) do we start to see some deviation between the exact solution and the first term in the asymptotic series. In order to illustrate this, we note that the asymptotic expansion of the evanescent waves also yields the asymptotic expansion of the travelling waves. For instance, from equation (12) we have $\operatorname{Re}\left[M_{\mathrm{a}}(q, \theta)\right]=(\cos q) / q$ and, if we subtract equation (69), we find that

$$
\operatorname{Re}\left[M_{\mathrm{a}}(q, \theta)^{\mathrm{tr}}\right]=\frac{\cos q}{q}-J_{0}(\rho) \frac{1}{|\zeta|}+\cdots
$$

Figure 6 shows that indeed for values of $q$ smaller than about a (dimensionless) wavelength the approximation (70), only using the two terms shown, starts to deviate from the exact value. 


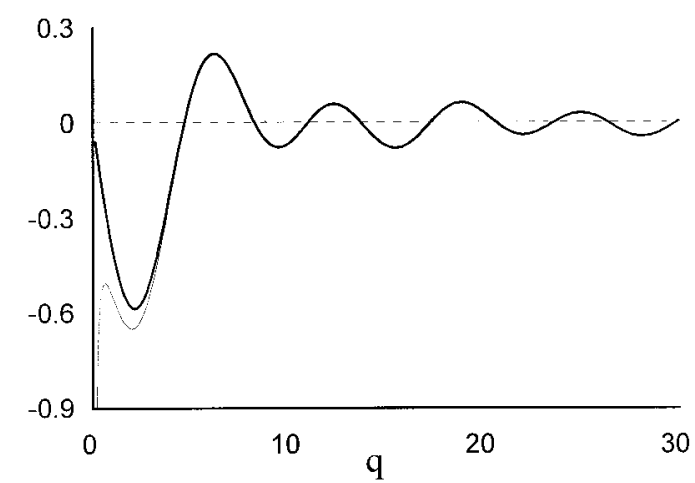

Figure 6. The exact value of $\operatorname{Re}\left[M_{\mathrm{a}}(q, \theta)^{\mathrm{tr}}\right]$ for $\theta=30^{\circ}(-)$, and its asymptotic approximation (-), as given by equation (70).

The asymptotic expansions for the other three functions can be obtained along similar lines. We find that

$$
\begin{aligned}
& M_{\mathrm{b}}(q, \theta)^{\mathrm{ev}}=-\sum_{k=0}^{\infty} \frac{(2 k) !}{k !}\left(-\frac{1}{2} \rho\right)^{k} J_{k-2}(\rho) \frac{1}{|\zeta|^{2 k+1}} \\
& M_{\mathrm{c}}(q, \theta)^{\mathrm{ev}}=-2 \sum_{k=0}^{\infty} \frac{(2 k+1) !}{k !}\left(-\frac{1}{2} \rho\right)^{k} J_{k-1}(\rho) \frac{1}{|\zeta|^{2 k+2}} \\
& M_{\mathrm{d}}(q, \theta)^{\mathrm{ev}}=-\sum_{k=0}^{\infty} \frac{(2 k+2) !}{k !}\left(-\frac{1}{2} \rho\right)^{k} J_{k}(\rho) \frac{1}{|\zeta|^{2 k+3}}
\end{aligned}
$$

and here we have used that Bessel functions of negative order are given by $J_{-n}(\rho)=(-1)^{n} J_{n}(\rho)$. It should be noted that these expansions are not entirely independent, since $M_{\mathrm{b}}(q, \theta)^{\mathrm{ev}}$ and $M_{\mathrm{c}}(q, \theta)^{\mathrm{ev}}$ are related as [11]

$$
|\zeta| M_{\mathrm{b}}(q, \theta)^{\mathrm{ev}}+\frac{1}{2} \rho M_{\mathrm{c}}(q, \theta)^{\mathrm{ev}}=-J_{2}(\rho),
$$

as can be derived from their integral representations, and as can be verified explicitly from equations (71) and (72). Also, for $M_{\mathrm{d}}(q, \theta)^{\mathrm{ev}}$ we have the relation

$$
3 M_{\mathrm{d}}(q, \theta)^{\mathrm{ev}}=-\frac{8 \pi}{k_{0}^{3}} \delta(\boldsymbol{r})+M_{\mathrm{a}}(q, \theta)^{\mathrm{ev}}-M_{\mathrm{b}}(q, \theta)^{\mathrm{ev}}-|\cot \theta| M_{\mathrm{c}}(q, \theta)^{\mathrm{ev}},
$$

as can be verified from equations (68) and (71)-(73) and recursion relations for Bessel functions. Since the asymptotic expansions only hold for $\zeta \neq 0$, the delta function in equation (75) is irrelevant here.

For a field point on the $z$ axis we have $\rho=0,|\zeta|=q$, and with $J_{k}(0)=\delta_{\mathrm{k}, 0}$ the asymptotic series reduce to

$$
\begin{aligned}
& M_{\mathrm{a}}(q, 0)^{\mathrm{ev}}=\frac{1}{q}, \\
& M_{\mathrm{b}}(q, 0)^{\mathrm{ev}}=M_{\mathrm{c}}(q, 0)^{\mathrm{ev}}=0, \\
& M_{\mathrm{d}}(q, 0)^{\mathrm{ev}}=-\frac{2}{q^{3}}
\end{aligned}
$$


(and the same for $\theta=\pi$ ). On the other hand, we observe from equations (47) and (53) that for $\theta=0$ we have $a_{n}(0)=c_{n}(0)=0$ for all $n$. Therefore, in equation (46), only the term $1 / q$ survives and the right-hand side of equation (52) reduces to $-2 / q^{3}$. Both equation (50) and equation (51) are proportional to $\sin \theta$, so these functions vanish identically for $\theta=0$. This shows that equations (76)-(78) are exact for all points on the $z$ axis $(\zeta \neq 0)$.

\section{New integral representations}

With equation (37), the coefficient functions $A_{n}(\theta)$ have the integral representation

$$
A_{n}(\theta)=\int_{0}^{|\cos \theta|} \mathrm{d} t\left(1-t^{2}\right)^{n}
$$

In this section we shall assume that $z \geq 0$, which will simplify the notation slightly. In equation (79) we make the substitution $t=\cos \theta^{\prime}$, which gives

$$
A_{n}(\theta)=\int_{\theta}^{\pi / 2} \mathrm{~d} \theta^{\prime}\left(\sin \theta^{\prime}\right)^{2 n+1} .
$$

When we substitute this expression in the series expansion (24) and change the summation and integration order, then the remaining series can be summed in closed form. We then find the new integral representation

$$
\operatorname{Re}\left[M_{\mathrm{a}}(q, \theta)^{\mathrm{tr}}\right]=-\int_{\theta}^{\pi / 2} \mathrm{~d} \theta^{\prime} J_{l}\left(q \sin \theta^{\prime}\right) .
$$

This remarkably simple form is an alternative to the representation (22), where the dependence on the field point coordinates $q$ and $\theta$ is far more cumbersome in appearance. We have verified numerically that both representations are indeed identical.

When we make the same change in variables in equation (48) we obtain for $a_{n}(\theta)$ the following:

$$
a_{n}(\theta)=\int_{0}^{\theta} \mathrm{d} \theta^{\prime}\left(\sin \theta^{\prime}\right)^{2 n+1}
$$

The series (46) for the evanescent part can then be summed in the same way, with the result

$$
M_{\mathrm{a}}(q, \theta)^{\mathrm{ev}}=\frac{1}{q}-\int_{0}^{\theta} \mathrm{d} \theta^{\prime} J_{l}\left(q \sin \theta^{\prime}\right),
$$

as an alternative to the representation (23). With the known integral

$$
\int_{0}^{\pi / 2} \mathrm{~d} \theta^{\prime} J_{l}\left(q \sin \theta^{\prime}\right)=\frac{1}{q}(1-\cos q),
$$


it follows that the sum of equations (81) and (83) equals $(\cos q) / q$, which is $\operatorname{Re}\left[M_{\mathrm{a}}(q, \theta)\right]$, as it should be. It is interesting to note that in these new representations the order of the Bessel functions is different from the order in the original representations.

The series for the travelling and evanescent parts of the function $M_{\mathrm{b}}(q, \theta)$ are determined by the same coefficient functions $A_{n}(\theta)$ and $a_{n}(\theta)$, and a similar calculation to above yields the new integral representations for the travelling and evanescent parts of this second function:

$$
\begin{aligned}
\operatorname{Re}\left[M_{\mathrm{b}}(q, \theta)^{\mathrm{tr}}\right] & =\sin ^{2} \theta \int_{\theta}^{\pi / 2} \mathrm{~d} \theta^{\prime} \frac{1}{\sin ^{2} \theta^{\prime}} J_{3}\left(q \sin \theta^{\prime}\right), \\
M_{\mathrm{b}}(q, \theta)^{\mathrm{ev}} & =-\sin ^{2} \theta\left(\frac{3}{q^{3}}+\frac{1}{2 q}+\frac{1}{8} q\right)+\sin ^{2} \theta \int_{0}^{\theta} \mathrm{d} \theta^{\prime} \frac{1}{\sin ^{2} \theta^{\prime}} J_{3}\left(q \sin \theta^{\prime}\right) .
\end{aligned}
$$

The travelling (evanescent) parts of the other two auxiliary functions are linear combinations of the travelling (evanescent) parts of the first two, as in equations (74) and (75), so their integral representations follow from the results given above.

\section{Conclusions}

We have studied the travelling and evanescent parts of the optical near field from the viewpoint of the electromagnetic Green's tensor. This tensor can be expressed in terms of four auxiliary functions, each of which splits into a travelling and an evanescent part. Since the imaginary part of the tensor is pure travelling, we only had to consider the real parts of the four functions. For each, a series expansion around the origin was obtained, both for the (real part of the) travelling parts and the evanescent parts. Here, the dimensionless radial distance $q$ to the origin is seen as the variable, with the polar angle $\theta$ fixed. Then the Taylor coefficients in these series expansions depend parametrically on $\theta$. It was shown that the Taylor coefficients could be expressed in terms of the four coefficient functions $A_{n}(\theta), C_{n}(\theta), a_{n}(\theta)$ and $c_{n}(\theta)$, which can be generated numerically by recursion. It was shown that all singular terms in the Green's tensor are accounted for by evanescent waves.

The series expansion around $q=0$ was complemented by a series expansion in $|\zeta|$ around $|\zeta|=0$ and an asymptotic expansion in $1 /|\zeta|$, which allows us to obtain the Green's tensor for all distances $q$. It appeared that there is a considerable region of overlap between the expansion around $q=0$ and the two expansions in $|\zeta|$.

We have derived an integral representation for the coefficient function $A_{n}(\theta)$ from the generating function for these coefficients. It was shown that new integral representations for the travelling and evanescent parts of the auxiliary functions could be derived, which are considerably simpler in their dependence on the field point coordinates $q$ and $\theta$ than the original defining integrals.

\section{References}

[1] Pohl, D. W., 1991, Advances in Optical and Electron Microscopy, edited by T. Mulvey and C. J. R. Sheppard (San Diego, California: Academic Press), p. 243.

[2] Pohl, D. W., and Courjon, D., (editors), 1993, Near Field Optics, Proceedings of the NATO Advanced Research Workshop on Near Field Optics, NATO Advanced Study Institute Series, Vol. 242 (Dordrecht: Kluwer). 
[3] Courjon, D., and Bainier, C., 1994, Rep. Prog. Phys., 57, 989.

[4] Paesler, M. A., and Moyer, P. J., 1996, Near Field Optics, Theory, Instrumentation, and Applications (New York: Wiley).

[5] Ohtsu, M. (editor), 1998, Near-Field Nano/Atom Optics and Technology (Berlin: Springer).

[6] Grattan, K. T. V., and Meggitt, B. T. (editors), 2000, Optical Fiber Sensor Technology, Fundamentals (Dordrecht: Kluwer).

[7] Born, M., and Wolf, E., 1980, Principles of Optics, seventh (expanded) edition (Oxford: Pergamon) appendix III, p. 890.

[8] Sherman, G. C., Stamnes, J. J., and Lalor, É., 1976, J. math. Phys., 17, 760.

[9] Setälä, T., Kaivola, M., and Friberg, A. T., 1999, Phys. Rev. E, 59, 1200.

[10] Lakhtakia, A., and Weiglhofer, W. S., 2000, J. Mod. Optics, 47, 759.

[11] Arnoldus, H. F., and Foley, J. T., 2002, J. opt. Soc. Am. A, 19, 1701.

[12] Baños, A., 1966, Dipole Radiation in the Presence of a Conducting Half-Space, International Series of Monographs in Electromagnetic Waves, Vol. 9, edited by A. L. Cullen, V. A. Fock and J. R. Wait (Oxford: Pergamon).

[13] TAI, C. T., 1971, Dyadic Green's Functions in Electromagnetic Theory (Scranton, Pennsylvania: Intext).

[14] Keller, O., 1996, Phys. Rep., 268, 85.

[15] Jackson, J. D., 1975, Classical Electrodynamics, second edition (New York: Wiley), p. 141.

[16] Keller, O., 1999, J. opt. Soc. Am. B, 16, 835.

[17] Arnoldus, H. F., 2001, J. opt. Soc. Am. B, 18, 547.

[18] van Bladel, J., 1961, IRE Trans. Antennas Propagation, 9, 563.

[19] Yaghjian, A. D., 1980, Proc. IEEE, 68, 248.

[20] van Bladel, J., 1991, Singular Electromagnetic Fields and Sources (Oxford: Clarendon), Chapter 3.

[21] Lakhtakia, A., and Mulholland, G. W., 1993, J. Res.: Nat. Inst. Stand. Techol., 98, 699.

[22] Mandel, L., and Wolf, E., 1995, Optical Coherence and Quantum Optics (Cambridge University Press), Section 3.2.4.

[23] Duffy, D. G., 2001, Green's Functions with Applications, Studies in Advanced Mathematics (Boca Raton, Florida: Chapman \& Hall-CRC), p. 104.

[24] Shchegrov, A. V., and Carney, P. S., 1999, J. opt. Soc. Am. A, 16, 2583.

[25] Berry, M. V., 2001, J. Mod. Optics, 48, 1535.

[26] Arnoldus, H. F., and Foley, J. T., 2002, Optics Commun., 207, 7. 